\title{
High prevalence of abdominal aortic aneurysm in patients with inguinal hernia
}

\author{
Angelos Megalopoulos ${ }^{\mathrm{a}}$, Orestis loannidis ${ }^{\mathrm{b}}$, Ioannis Varnalidisc, Maria Ntoumparab , Lina Tsigriki ${ }^{\mathrm{b}}$, Konstantinos Alexandris ${ }^{\mathrm{b}}$, \\ Christiana Anastasiadou ${ }^{b}$, Parpoudi Stylianii ${ }^{b}$, George Paraskevas ${ }^{d}$, Ioannis Mantzoros ${ }^{b}$
}

\begin{abstract}
Aims. There is increased prevalence of inguinal hernia $(\mathrm{IH})$ in patients with abdominal aortic aneurysm (AAA). As there is limited data on AAA in patients with $\mathrm{IH}$ our objective was to examine the prevalence of AAA in such patients.

Methods. We prospectively examined 185 consecutive patients for AAA who presented to our department for surgical repair of an $\mathrm{IH}$. All patients were referred for ultrasound of the abdominal aorta. An AAA was considered to be present when the distal diameter of the abdominal aorta was over $3 \mathrm{~cm}$. Patients with no AAA were followed annually with an abdominal ultrasound for 5 years.

Results. Out of the 185 patients (179 males, 6 females) aged from 35-81 (mean 58.6 years), AAA initially appeared in 28 patients with a mean age 61.2 years old. The range of the aortic distal diameter was between 3.4 and $8.1 \mathrm{~cm}$ with a mean diameter of $4.8 \mathrm{~cm}$ in patients with AAA. The prevalence of the AAA was increased in smokers, with hypertension and with bilateral and direct hernia. At the end of the 5 years follow-up, 16 more patients had developed an AAA of mean diameter $3.2 \mathrm{~cm}(3-4.1 \mathrm{~cm})$, increasing the prevalence of AAA to $27.7 \%$.

Conclusion. There is an increased prevalence of AAA in patients with $\mathrm{IH}$, especially in smokers, with hypertension and with bilateral and/or direct hernia. Hence, periodic ultrasonound may play an important role in screening and early diagnosis of AAA in these patients.
\end{abstract}

Key words: abdominal aortic aneurysm, aorta, inguinal hernia, ultrasound, screening

Received: May 26, 2018; Revised: October 29, 2018; Accepted: December 5, 2018; Available online: January 30, 2019 https://doi.org/10.5507/bp.2018.077

(c) 2019 The Authors. This is an open access article licensed under the Creative Commons Attribution License (https://creativecommons.org/licenses/by/4.0/).

aDepartment of Cardiothoracic and Vascular Surgery, General Regional Hospital "George Papanikolaou", Thessaloniki, Greece 'Fourth Surgical Department, Medical School, Aristotle, University of Thessaloniki, Thessaloniki, Greece cPlastic Surgery Department, General Regional Hospital "George Papanikolaou", Thessaloniki, Greece ${ }^{d}$ Department of Anatomy, Medical School, Aristotle University of Thessaloniki, Thessaloniki, Greece Corresponding author: Orestis loannidis, e-mail: telonakos@hotmail.com

\section{INTRODUCTION}

Abdominal aortic aneurysm (AAA) is defined as a permanent focal dilation of the abdominal aorta with at least a $50 \%$ increase over normal arterial diameter ${ }^{1}$. Most cases of AAA are encountered in the infrarenal portion of the abdominal aorta and end above the iliac arteries $^{2}$. AAA has been increasing in the last years and large screening studies have suggested a prevalence of around $5 \%$ in men over the age of $50\left(\right.$ ref. $\left.^{2-4}\right)$. The vast majority of AAA remain asymptomatic unless they rupture; a lethal catastrophic event associated with an important mortality rate even when emergency surgical interventions are performed on time $e^{2,5,6}$.

Early detection and elective surgery may decrease the high mortality and prevent death from ruptured aneurysms of the abdominal aorta, as the mortality rate in emergency operations is extremely high ${ }^{2,6,7}$. Thus, it is of the utmost importance to establish a screening method in subjects who are at high risk $^{8}$. Screening people at increased risk may be cost effective if the number of people who require screening is lower and so acceptable. It has been demonstrated that ultrasound examination is a relatively inexpensive, noninvasive and almost $100 \%$ accurate method in identifying and following patients with AAA and is considered to be the appropriate screening tool in high risk patients ${ }^{2,8,9}$.

Many studies search for the etiology of AAA, analyzing subpopulations or patients and several risk groups have been identified ${ }^{9}$. Age, male gender, smoking, hypertension, relatives of patients with AAA, atherosclerosis and peripheral arterial obstructive disease, pulmonary emphysema, diabetes mellitus, high cholesterol levels, have been associated with the development of aneurysms of the abdominal aorta in previous studies ${ }^{8-11}$. In addition, an increased prevalence of inguinal hernia $(\mathrm{IH})$ in patients with confirmed AAA has been reported and men with a history of IH are considered at high risk of AAA, especially if they smoke ${ }^{10,12-17}$.

The association between $\mathrm{IH}$ and abdominal aortic aneurysm may be attributed to elastin and collagen fiber abnormalities and disorders ${ }^{8,18-22}$. Alterations in the levels of collagenase, elastase and antiproteases may be important components in the pathophysiology of both diseases. Smoking interferes with the protease / antiprotease system and is thought to enhance pathophysiologic mechanisms.

The aim of the present study is to examine the prevalence of AAA in patients with $\mathrm{IH}$ and the relation between 
Table 1. Characteristics of patients with inguinal hernia regarding the presence of AAA.

\begin{tabular}{lccc}
\hline & $\begin{array}{c}\text { Patients } \\
\text { with AAA }\end{array}$ & $\begin{array}{c}\text { Patients } \\
\text { without AAA }\end{array}$ & $P$ \\
\hline $\mathrm{n}$ & 28 & 157 & $<0.001$ \\
Age & $61.2 \pm 3.1$ & $57.4 \pm 2.6$ & 0.001 \\
Bilateral Inguinal Hernia & 11 & 20 & $<0.001$ \\
Direct Inguinal Hernia & 15 & 30 & $<0.001$ \\
Smoking & 23 & 70 & 0.002 \\
Hypertension & 18 & 51 & 0.197 \\
Hypercholesterolemia & 16 & 66 & 0.151 \\
Diabetes Mellitus & 5 & 14 & 0.703 \\
Peripheral Arterial Disease & 5 & 33 & \\
\hline
\end{tabular}

these two diseases and to define a new high risk population for AAA who could benefit from screening.

\section{MATERIALS AND METHODS}

From January 1999 until March 2001, 185 consecutive patients (179 men and 6 women) from 35 to 81 years of age (mean 58.6 years), who visited our department for IH repair, were analyzed in our study. Informed consent was obtained from all participants. The study was reviewed and approved by the institutional scientific board and study was performed according to the Helsinki Declaration. All patients were interviewed and examined both by an experienced general surgeon and vascular surgeon of our department and an ultrasonography of the abdominal aorta was performed by a radiologist with extensive experience in the field.

In the interview several risk factors for the development of AAAs were assessed (age, smoking, hypertension). A complete blood test was performed on all patients. The physical examination included bilateral upper extremity blood pressure measurement, carotid arteries and abdominal auscultation, femoral, popliteal and pedal (dorsalis pedis and posterior tibial) palpation, and search for a pulsatile abdominal mass with the patient in the supine position with his knees slightly flexed. The IHs were reexamined and classified as unilateral or bilateral.

Patients were defined as smokers if they had been smoking at least 10 cigarettes per day, until admission or if there was a history of at least 20 years nicotine given up not more than 10 years ago. Hypertension was defined as $\mathrm{SP}>140 \mathrm{mmHg}$ or DP>90 $\mathrm{mmHg}$ or if the patient was receiving antihypertensive treatment. Hypercholesterolemia was defined as total cholesterol levels $\geq 200 \mathrm{mg} / \mathrm{dL}$, LDL levels $\geq 100 \mathrm{mg} / \mathrm{dL}$ or the patient was under medical treatment. The consensus criteria for diabetes mellitus were used (random blood glucose $\geq 126 \mathrm{mg} / \mathrm{dL}$ ) or if the patient was treated by a diabetologist. Arterial occlusive disease (AOD) was defined as a patient's surgical history positive for a previous intervention or medical history positive for claudication, rest pain or gangrene.

Ultrasonography of the abdominal aorta was performed using a SONOLINE ANTARES ultrasound (SIEMENS, Siemens Medical Solutions, Mountain View,
USA) and a 3.5 MHz transducer in transverse and longitudinal projections. All patients were in the supine position. The suprarenal aortic diameter was measured $\sim 1 \mathrm{~cm}$ distal to the origin of the superior mesenteric artery, just above the level of the renal arteries. The infrarenal aortic diameter was measured at the level of the maximum diameter of the abdominal aorta below the renal arteries. An AAA was diagnosed when the aortic diameter was $3 \mathrm{~cm}$ or more. Patients with no AAA were followed annually with an ultrasound of the abdominal aorta for 5 years. Those with aneurysms between 3 and $4.9 \mathrm{~cm}$ were followed with a CT-scan every 6 months and AAAs larger than $5 \mathrm{~cm}$ were electively repaired, taken into consideration other surgical or anesthetic contraindications.

In addition, the patients were divided in 3 groups according to the time period they had the IH. The groups were as follows regarding the patients at the time they were first examined: Group I consisted of patients who had a hernia for more than 10 years, group II consisted of those who had a hernia more than 5 years but less than 10 and group III consisted of patients with a hernia for less than 5 years. After the five years follow up the groups were changed as follows: Group Ia consisted of patients who had been diagnosed with a hernia for more than 15 years, group IIa consisted of those who had a hernia more than 10 years but less than 15 and group IIIa consisted of patients with a hernia for more than 5 and less than 10 years.

The data was computerized for statistical analysis. The chi-square test ( $\chi^{2}$ test) was used to compare nominal variables and Student's t test for continues variables. Logistic regression was used in order to calculate the odds ratio. The confidence interval was $95 \%$, and a difference was considered statistically significant at $P<0.05$. The statistical analysis was performed using SPSS (Statistical Package for Social Sciences, version 14.0).

\section{RESULTS}

The patients were predominantly male ( $n=160,98 \%$ ). Aneurysm of the abdominal aorta was diagnosed in 28 patients, all males, with a history of IH (15.1\%). The mean age at diagnosis was $61.2 \pm 3.1$ years. The mean diameter of AAA was $4.8 \mathrm{~cm}$ (3.4-8.1). Nine patients had an aneu- 
rysm ranging from $3-4 \mathrm{~cm}, 8$ patients had an aneurysm with diameter $4-5 \mathrm{~cm}$ and 11 patients had an aneurysm larger than $5 \mathrm{~cm}$. The mean age of patients without an AAA was $57.4 \pm 2.6$ years. The mean age of patients with AAA was significantly higher than those without AAA $(P<0.001)$. The prevalence of AAA clearly increases with age.

Regarding the time period the patients had the IH, 102 patients $(55.1 \%)$ belonged in group I of whom 19 had an AAA (18.6\%), 51 patients (27.5\%) belonged to group II of whom 7 had an AAA (13.7\%), and 32 (17.4\%) belonged to group III of whom 2 had an AAA (6.3\%). No statistically significant difference was found $(P=0.222)$. All patients with an AAA greater than $4 \mathrm{~cm}$ belonged to group I.

Out of the 185 patients, 31 patients presented bilateral IH (16.7\%), while the 11 patients from the 28 with AAA had a bilateral IH (39.2\%). In the group of the patients with bilateral IHs the prevalence of AAA was $35.5 \%$. Out of the 31 patients with a bilateral $\mathrm{IH}, 23$ patients (74.2\%) belonged to group I from which 9 patients presented with AAA (39.1\%) and 8 patients (25.8\%) belonged to group II from which 2 patients presented with AAA (25\%). There was a statistically significant correlation between bilateral IH and AAA $(P=0.001)$ and patients with bilateral $\mathrm{IH}$ are in increased risk of developing an AAA. Specifically, patients with bilateral IH have a 4.4 times increased probability of developing an AAA (odds ratio 4,432, 95\% C.I.: $1,817-10,813, P=0.001$ ).

Out of the 185 patients, 140 had an indirect hernia (75.7\%) and 45 had a direct hernia (24.3\%). Of the 28 patients with AAA 13 had indirect hernia (46.4\%) and 15 had a direct hernia (53.6\%). There was a statistically significant correlation between direct IH and AAA $(P<0.001)$ and patients with direct IH are in increased risk of developing an AAA. Specifically, patients with direct IH had a 4.9 times increased probability of developing an AAA (odds ratio 4,885, 95\% C.I.:2,103-11,343, $P<0.001$ ) compared to patients with indirect IH.

Smokers composed 93 patients of all patients (50.2\%), while 23 patients were smokers in the AAA group $(82.1 \%)$. In the group of smokers the prevalence of AAA was $24.7 \%$. There was a statistically significant correlation between smoking and AAA $(P<0.001)$ and smokers are in increased risk of developing an AAA. Specifically, smokers have a 5.7 times increased probability of developing an AAA (odds ratio 5,717, 95\% C.I.:2,068-15,808, $P<0.001$ ).

Hypertension was diagnosed in 69 of all patients (37.3\%), while of the patients with AAA 18 patients had hypertension $(64.3 \%)$. In the group of patients with hypertension the prevalence of AAA was $26 \%$. There was a statistically significant correlation between hypertension and AAA $(P=0.001)$ and patients with hypertension are in increased risk of developing an AAA. Specifically, patients with hypertension have a 3.7 times increased possibility of developing an AAA (odds ratio 3,741, 95\% C.I.: 1612-8,684, $P=0.002$ ).

Hypercholesterolemia was diagnosed in 82 of all patients (44.3\%) while of the patients with AAA 16 had hypercholesterolemia $(57.1 \%)$ and in patients without AAA, hypercholesterolemia was found in $42 \%$. In the group of patients with hypercholesterolemia the prevalence of AAA was $19.5 \%$. There was no statistically significant correlation between hypercholesterolemia and AAA $(P=0.197)$ and patients with hypercholesterolemia are not in increased risk of developing an AAA.

Diabetes mellitus was diagnosed in 19 of all patients (10.2\%) while of the patients with AAA 5 patients had diabetes $(17.8 \%)$ and in patients without AAA, diabetes was present in $8.9 \%$. In the patients with diabetes, the prevalence of AAA was $26.3 \%$. There was no statistically significant correlation between diabetes and AAA $(P=0.151)$ and patients with diabetes were not in increased risk of developing an AAA.

Regarding the presence of peripheral arterial occlusive disease, in patients with AAA, 5 had AOD (17.8\%) while in patients without AAA, 33 had AOD (21\%). In the patients with AOD the prevalence of AAA was $13.1 \%$. There was no statistically significant correlation between AOD and AAA $(P=0.703)$ and patients with AOD were not in increased risk of developing an AAA.

A detailed presentation of patients with and without AAAs at the time of presentation according to studied parameters is shown in Table 1.

The 157 patients who had no AAA were scheduled for annual ultrasound of the abdominal aorta for 5 years. Twenty six patients were lost on follow up and 16 of the remaining $131(12.2 \%)$ presented an AAA at the end of the 5 years follow up with a mean diameter $3.4 \mathrm{~cm}$ (3.1-4.2 $\mathrm{cm})$. Regarding the time period the patients had the $\mathrm{IH}$, 68 patients $(51.9 \%)$ belonged to group Ia where 8 developed an AAA (11.7\%), 39 patients (29.8\%) belonged to group IIa in which 5 developed an AAA (12.8\%), and 24 (18.3\%) belonged to group IIIa in which 3 developed an AAA (12.5\%). No statistically significant difference was found $(P=0.986)$

At the end of the five year follow-up period, the overall prevalence of AAA in patients with IH was at least $27.7 \%$ $(n=44)$. Aneurysms were much more prevalent in men $(n=44)$ than in women $(n=0)$. Eleven patients $(5.9 \%)$ had an aneurysm greater than $5 \mathrm{~cm}$. No significant differences were observed regarding the years the patients had the IH and the presence of AAA either in the initial presentation or at the end of the 5 years follow up.

\section{DISCUSSION}

In the 80 s a new theory for the development of AAA was developed $^{12,18-22}$. It suggested that AAA is a special condition that is not caused by arteriosclerosis, although AAA can co-exist with arteriosclerosis. Comparing patients suffering from AAA with others suffering from peripheral AOD, the authors found remarkable differences, which helped to distinguish the two diseases and support the theory that the occurrence of AAA and its rupture is the result of systematic elastin and collagen fiber degeneration $^{12,18-22}$. The important observations of this study on patients with AAA can be summarized as following: they have first degree relatives with AAA, negative rhesus factor, and 0 blood type at an increased percentage; patients 
with AAA are about 10 years younger compared to those with peripheral AOD, they are predominately males and they do not suffer from peripheral AOD or present with ischemia of the lower extremities. They present "arteriomegaly", meaning aneurysmatic dilation of other blood vessels too except for the abdominal aorta, and they present with an increased prevalence of emphysema and increased prevalence of IH or any hernia ${ }^{12,18-22}$. The last observation of increased incidence of $\mathrm{IH}$ in patients with AAA compared to those with AOD is also supported by multiple studies ${ }^{13-16}$.

The reported incidence of IHs ranges from 19\% to $41 \%$ in patients with AAA, whereas in patients with AOD disease the incidence varies between 5\% and 19\% (ref. ${ }^{13-}$ ${ }^{16,23-25}$ ). Also, the frequency of the development of postoperative hernia is much higher in patients with AAA. Raffeto et al. ${ }^{26}$ reported an incidence of $28.2 \%$ of postoperative hernia in patients with AAA and $11 \%$ in patients with AOD, and according to Tagaki et al. ${ }^{27}$, patients with AAA have approximately 3 times greater incidence of IHs and incisional hernias compared to patients with AOD.

The studies presented above are based on the comparison between patients with AAA and patients with AOD. However, there is little data in the literature regarding the reverse correlation, whether in patients with diagnosed IH, AAA is more common, if it occurs before the diagnosis and the surgical treatment of hernia or during its natural course. Likewise, there is no indication of whether AAA is more common in patients with bilateral IH.

According to the current study there seems to be an increased prevalence of AAA in patients with diagnosed IH. Few studies have investigated the prevalence of AAA in patients with a history of IH. Pleumeekers et al. ${ }^{10}$ examined whether men aged 55 years or older with a history of IH were at increased risk of having an AAA. The prevalence of AAA was $12.2 \%$, so there is an increased risk, notably if the patients are cigarette smokers ${ }^{10}$. Antoniou et al. ${ }^{17}$ also reported an increased prevalence of AAA in over 55-year-old men undergoing IH repair that could justify screening. In addition, patients with $\mathrm{IH}$ had an increased aortic diameter, indicating that IHs make the development of an AAA more likely in the future ${ }^{17}$. In the present study an increased prevalence of AAA in patients with $\mathrm{IH}$ was observed. Compared to the suggested prevalence of AAA of $5 \%$ in men over 50 years old we observed a prevalence of $15.1 \%$ in patients with $\mathrm{IH}$ at the time of presentation for surgery which increased to at least $27.7 \%$ at the end of the 5 years follow up, as $12.2 \%$ of the patients submitted to follow up presented with an AAA. No significant differences were observed regarding the years the patients had the IH and the presence of AAA both in the initial presentation and at the end of the 5 years follow up. So the patients with IH are both in an increased risk of an AAA at the time of a doctor visit and developing an AAA during follow up. Patients who smoke, have hypertension and bilateral or direct $\mathrm{IH}$ are at more increased risk for an AAA compared to patients with a one side indirect $\mathrm{IH}$, who are not smokers and have normal blood pressure. In contrast, Anderson et al. ${ }^{27}$ demonstrated that AAA is no more common in over 65-year-old men with IH, compared to the incidence of AAA in UK screening programmes which select patients with only age and sex criteria. As a result, they concluded preoperatively selective screening is of no great value ${ }^{27}$. However, AAA occur in average 13 years after the presentation of $\mathrm{IH}$ and therefore, a single ultrasonographical examination at the time of hernia repair would be inadequate ${ }^{28}$.

Abdominal aortic aneurysms are an important and preventable cause of death. Early detection attempted by screening programs may prevent death from rupture ${ }^{29}$. Several high risk factors are smoking, hypertension, AOD, relatives with AAA (ref. ${ }^{15,16,23,30-33}$ ), but currently, age and sex are the only risk factors that justify screening ${ }^{34}$. In the current study a significant correlation between the presence of AAA and smoking, hypertension and bilateral or direct IH was observed. Other studies have also demonstrated an increased prevalence of AAA in patients with $\mathrm{IH}\left(\right.$ ref. $\left.^{10,17}\right)$. From the current data there seems to be a correlation between AAA and IH. Ultrasonographic screening is important as far as the early diagnosis of AAA is concerned ${ }^{35}$.

The development of AAA and its rupture is a result of the disruption of the normal levels and ratio of elastin/ elastase and collagen/collagenase ${ }^{12}$. Elastase and collagenase are proteolytic enzymes. The decrease of elastin in the aortic wall is responsible for the occurrence of AAA and the decrease of collagen is responsible for its rupture. The role of these enzymes has been confirmed in several studies $^{13,15,36-42}$. The abdominal wall presents loss of collagen fibril assembly ${ }^{32}$; a systemic or constitutive increase in collagen synthesis might have such a result, while a twofold increase in the concentrations of type III collagen in skin fibroblasts in patients with IHs, predisposing them to the development of recurrent or incidental inguinal herniation ${ }^{23}$. Furthermore, the increased proteolytic activity may contribute to the higher frequency of IHs in patients with AAA ( ref. $^{13}$ ). It has also been demonstrated that there is a correlation with AAA and increased rates of elastin and homocysteine in blood serum ${ }^{43,44}$.

\section{CONCLUSION}

According to this study there is an increased prevalence of AAA in patients with diagnosed $\mathrm{IH}$, especially in smokers, with hypertension and with bilateral and direct $\mathrm{IH}$. Hence, periodic ultrasonography may play an important role in screening and early diagnosis of AAA in patients with IH. However, the sample in this study can be considered small and we need more patients, especially patients with bilateral or direct IH, as large cohort studies have failed to show an association between AAA and IH $\left(\right.$ ref. $^{45}$ ), while others have shown an increased incidence of IH in patients with thoracic aortic aneurysm ${ }^{46}$. To conclude, the prevalence of AAA in patients with IH must be further examined ${ }^{47}$, as the need of selective screening programs for those patients is disputable ${ }^{23,32}$. 


\section{ABBREVIATIONS}

AAA: abdominal aortic aneurysm, IH: inguinal hernia.

Author contributions: All authors helped to perform the research; MA, IO, VI, PG, MI collected the data. IO, NM, TL, AK, AC, PS analyzed and interpreted the data. MA, IO, VI, PG, MI designed and supervised the study. MA, IO, VI, PG, MI significantly contributed to the literature review and linguistic formatting and correction of the manuscript. IO, NM, TL, AK, AC, PS, revised it critically for important intellectual content. MA, IO, NM, TL, drafted the manuscript and were responsible for final proof reading. All authors have read and approved the final version to be published.

Conflict of interest statement: None declared.

Presented: $3^{\text {rd }}$ PanHellenic hernia congress, 10/03/200612/03/2006, Thessaloniki Greece

\section{REFERENCES}

1. Ruiz CE, Zhang HP, Douglas JT, Zuppan CW, Kean CJ. A novel method for treatment of abdominal aortic aneurysms using percutaneous implantation of a newly designed endovascular device. Circulation 1995;91(9):2470-7.

2. Crawford CM, Hurtgen-Grace K, Talarico E, Marley J. Abdominal aortic aneurysm: an illustrated narrative review. J Manipulative Physio Ther 2003;26(3):184-95. Review. PubMed PMID: 12704311.

3. Howell MH, Zaqqa M, Villareal RP, Strickman NE, Krajcer Z. Endovascular exclusion of abdominal aortic aneurysms: initial experience with stent-grafts in cardiology practice. Tex Heart Inst J 2000;27(2):136-45

4. Pleumeekers HJ, Hoes AW, van der Does E, van Urk H, Grobbee DE. Epidemiology of abdominal aortic aneurysms. Eur J Vasc Surg 1994;8(2):119-28. Review.

5. Ioannidis O, Trellopoulos G, Tamouridis G, Konstantinidis K, Megalopoulos A. A single-centre experience of the treatment of ruptured abdominal aortic aneurysms: clinical and anatomic characteristics of open versus endovascular repair. Int Angiol 2012;31(4):386-92

6. Thompson RW, Geraghty PJ, Lee JK. Abdominal aortic aneurysms: basic mechanisms and clinical implications. Curr Probl Surg 2002;39(2):110-230. Review.

7. Singh K, Bønaa KH, Jacobsen BK, Bjørk L, Solberg S. Prevalence of and risk factors for abdominal aortic aneurysms in a population-based study: The Tromsø Study. Am J Epidemiol 2001;154(3):236-44.

8. Sakalihasan N, Limet R, Defawe OD. Abdominal aortic aneurysm. Lancet 2005;365(9470):1577-89. Review.

9. Upchurch GR Jr, Schaub TA. Abdominal aortic aneurysm. Am Fam Physician 2006;73(7):1198-204. Review.

10. Pleumeekers HJ, De Gruijl A, Hofman A, Van Beek AJ, Hoes AW. Prevalence of aortic aneurysm in men with a history of inguinal hernia repair. Br J Surg 1999;86(9):1155-8.

11. Anderson $O$, Shiralkar S. Prevalence of abdominal aortic aneurysms in over 65-year-old men with inguinal hernias. Ann R Coll Surg Engl 2008;90(5):386-8. doi: 10.1308/003588408X285937

12. Reilly JM, Tilson MD. Incidence and etiology of abdominal aortic aneurysms. Surg Clin North Am 1989;69(4):705-11. Review.

13. Cannon DJ, Casteel L, Read RC. Abdominal aortic aneurysm, Leriche's syndrome, inguinal herniation, and smoking. Arch Surg 1984;119(4):387-9.

14. Lehnert $B$, Wadouh F. High coincidence of inguinal hernias and abdominal aortic aneurysms. Ann Vasc Surg 1992;6(2):134-7.

15. Hall KA, Peters B, Smyth SH, Warneke JA, Rappaport WD, Putnam CW, Hunter GC. Abdominal wall hernias in patients with abdominal aortic aneurysmal versus aortoiliac occlusive disease. Am J Surg 1995;170(6):572-5; discussion 575-6.
16. Adye B, Luna G. Incidence of abdominal wall hernia in aortic surgery. Am J Surg 1998;175(5):400-2.

17. Antoniou GA, Giannoukas AD, Georgiadis GS, Antoniou SA, Simopoulos C, Prassopoulos P, Lazarides MK. Increased prevalence of abdominal aortic aneurysm in patients undergoing inguinal hernia repair compared with patients without hernia receiving aneurysm screening. J Vasc Surg 2011;53(5):1184-8. doi: 10.1016/j. jvs.2010.11.053.

18. Ailawadi G, Eliason JL, Upchurch GR Jr. Current concepts in the pathogenesis of abdominal aortic aneurysm. J Vasc Surg 2003;38(3):584-8. Review.

19. Tilson MD, Seashore MR. Human genetics of the abdominal aortic aneurysm. Surg Gynecol Obstet 1984;158(2):129-32.

20. Tilson MD, Seashore MR. Fifty families with abdominal aortic aneurysms in two or more first-order relatives. Am J Surg 1984;147(4):5513.

21. Tilson MD. Histochemistry of aortic elastin in patients with nonspecific abdominal aortic aneurysmal disease. Arch Surg 1988;123(4):503-5.

22. Tilson MD, Dang C. Generalized arteriomegaly. A possible predisposition to the formation of abdominal aortic aneurysms. Arch Surg 1981;116(8):1030-2.

23. Musella M, Milone F, Chello M, Angelini P, Jovino R. Magnetic resonance imaging and abdominal wall hernias in aortic surgery. J Am Coll Surg 2001;193(4):392-5.

24. Papadimitriou D, Pitoulias G, Papaziogas B, Koutsias S, Vretzakis G, Argiriadou $\mathrm{H}$, Papaziogas $\mathrm{T}$. Incidence of abdominal wall hernias in patients undergoing aortic surgery for aneurysm or occlusive disease. Vasa 2002;31(2):111-4.

25. Raffetto JD, Cheung Y, Fisher JB, Cantelmo NL, Watkins MT, Lamorte WW, Menzoian JO. Incision and abdominal wall hernias in patients with aneurysm or occlusive aortic disease. J Vasc Surg 2003;37(6):1150-4.

26. Takagi $H$, Sugimoto $M$, Kato T, Matsuno $Y$, Umemoto T. Postoperative incision hernia in patients with abdominal aortic aneurysm and aortoiliac occlusive disease: a systematic review. Eur J Vasc Endovasc Surg 2007;33(2):177-81.

27. Anderson $\mathrm{O}$, Shiralkar S. Prevalence of abdominal aortic aneurysms in over 65-year-old men with inguinal hernias. Ann R Coll Surg Engl 2008;90(5):386-8. doi: 10.1308/003588408X285937

28. Wright DM, O'Dwyer PJ. Prevalence of aortic aneurysm in men with a history of inguinal hernia repair. Br J Surg 2000;87(1):123-4.

29. Collin J. Screening for abdominal aortic aneurysms. Br J Surg 1985;72(11):851-2.

30. Bennett HF, Balfe DM. MR imaging of the peritoneum and abdominal wall. Magn Reson Imaging Clin N Am 1995;3(1):99-120. Review.

31. Stevick CA, Long JB, Jamasbi B, Nash M. Ventral hernia following abdominal aortic reconstruction. Am Surg 1988;54(5):287-9.

32. Holland AJ, Castleden WM, Norman PE, Stacey MC. Incisional hernias are more common in aneurysmal arterial disease. Eur J Vasc Endovasc Surg 1996;12(2):196-200.

33. Schrenk P, Bettelheim P, Woisetschläger R, Rieger R, Wayand WU. Metabolic responses after laparoscopic or open hernia repair. Surg Endosc 1996;10(6):628-32.

34. Ashton HA, Buxton MJ, Day NE, Kim LG, Marteau TM, Scott RA, Thompson SG, Walker NM; Multicentre Aneurysm Screening Study Group. The Multicentre Aneurysm Screening Study (MASS) into the effect of abdominal aortic aneurysm screening on mortality in men: a randomised controlled trial. Lancet 2002;360(9345):1531-9.

35. Fleming C. Screening and management of abdominal aortic aneurysm: the best evidence. Am Fam Physician 2006;73(7):1157-8.

36. Powell J, Greenhalgh RM. Cellular, enzymatic, and genetic factors in the pathogenesis of abdominal aortic aneurysms. J Vasc Surg 1989;9(2):297-304.

37. Busuttil RW, Abou-Zamzam AM, Machleder HI. Collagenase activity of the human aorta. A comparison of patients with and without abdominal aortic aneurysms. Arch Surg 1980;115(11):1373-8.

38. Satta J, Juvonen T, Haukipuro K, Juvonen M, Kairaluoma MI. Increased turnover of collagen in abdominal aortic aneurysms, demonstrated by measuring the concentration of the aminoterminal propeptide of type III procollagen in peripheral and aortal blood samples. J Vasc Surg 1995;22(2):155-60.

39. Anderson DW, Edwards TK, Ricketts MH, Kuivaniemi H, Tromp G, Stolle CA, Deak SB, Boyd CD. Multiple defects in type III collagen 
synthesis are associated with the pathogenesis of abdominal aortic aneurysms. Ann N Y Acad Sci 1996;800:216-28.

40. Friedman DW, Boyd CD, Norton P, Greco RS, Boyarsky AH, Mackenzie JW, Deak SB. Increases in type III collagen gene expression and protein synthesis in patients with inguinal hernias. Ann Surg 1993;218(6):754-60.

41. Langer S, Christiansen J. Long-term results after incisional hernia repair. Acta Chir Scand 1985;151(3):217-9.

42. Cannon DJ, Read RC. Blood elastolytic activity in patients with aortic aneurysm. Ann Thorac Surg 1982;34(1):10-5.

43. Lindholt JS, Heickendorff L, Henneberg EW, Fasting H. Serum-elastinpeptides as a predictor of expansion of small abdominal aortic aneurysms. Eur J Vasc Endovasc Surg 1997;14(1):12-6.

44. Brunelli T, Prisco D, Fedi S, Rogolino A, Farsi A, Marcucci R, Giusti
B, Pratesi C, Pulli R, Gensini GF, Abbate R, Pepe G. High prevalence of mild hyperhomocysteinemia in patients with abdominal aortic aneurysm. J Vasc Surg 2000;32(3):531-6.

45. Henriksen NA, Sorensen LT, Jorgensen LN, Lindholt JS. Lack of association between inguinal hernia and abdominal aortic aneurysm in a population-based male cohort. Br J Surg 2013;100(11):1478-82. doi: 10.1002/bjs.9257

46. Olsson C, Eriksson P, Franco-Cereceda A. Association between thoracic aortic disease and inguinal hernia. J Am Heart Assoc 2014;3(4). pii: e001040. doi: 10.1161/JAHA.114.001040

47. Bosanquet DC, Twine CP, Williams IM. Lack of association between inguinal hernia and abdominal aortic aneurysm in a populationbased male cohort (Br J Surg 2013; 100: 1478-1482). Br J Surg 2014;101(3):290-1. doi: 10.1002/bjs.9428 